\title{
The Diaphragm: Contractile Properties and Fatigue
}

\author{
Dudley F. Rochester \\ University of Virginia School of Medicine, Charlottesville, Virginia 22908
}

\section{Introduction}

The diaphragm is a striated skeletal muscle controlled by voluntary and automatic neural pathways. Its primary function is the act of breathing, specifically the inspiratory phase. The diaphragm responds to neural drive and work load much like nonrespiratory striated skeletal muscles, and its functional characteristics are primarily determined by its contractile properties. However, diaphragmatic contraction is modified by particular constraints imposed by respiratory system mechanics and control mechanisms. For example, Fenn (1) noted that the diaphragm contracts rhythmically against elastic and resistive forces, and that with each relaxation the diaphragm returns to a relatively constant resting position, determined by the balance of lung and chest wall recoil forces. Limb muscles also contract rhythmically during locomotion, but they must overcome predominantly inertial forces, and they have no special resting position. The most prominent influence on the diaphragm is the fact that, like the heart, it must contract in repetitive rhythmic fashion for life.

\section{Muscle fiber types and innervation}

Breathing is endurance work, and the muscle fiber composition of the diaphragm is well suited to the task (2). $\sim 55 \%$ of the fibers in adult human diaphragm are of the slow twitch, oxidative type, which are highly resistant to fatigue. $\sim 25 \%$ are of the fast twitch, oxidative glycolytic type, which, though intermediate, are relatively resistant to fatigue. The remaining $20 \%$ of the fibers are of the fast twitch glycolytic variety, which are susceptible to fatigue. In dogs and mice fast twitch glycolytic fibers are absent, whereas in other species they compose 20 $40 \%$ of the diaphragm muscle fiber population (2). In general, slow twitch oxidative fibers are relatively well endowed with capillaries, myoglobin, mitochondria, and isoenzymes that favor aerobic metabolism, whereas fast twitch glycolytic fibers have less myoglobin and mitochondria, and isoenzymes that favor anaerobic glycolysis $(3,4)$. Slow twitch fibers have low, and fast twitch fibers have high myosin ATPase activity, which accounts for the difference in contractile properties $(3,4)$. In most species, including the adult human, $75 \%$ or more of diaphragm muscle fibers have good to excellent endurance characteristics.

Neural control of the diaphragm is mediated via the phrenic nerve. Phrenic motoneurones lie in the cervical spinal cord, at segments 3-5 in humans, 4-6 in the cat, and 5-7 in

Dr. Rochester is the E. Cato Drash Professor of Medicine. Received for publication 6 February 1985.

J. Clin. Invest.

(c) The American Society for Clinical Investigation, Inc.

0021-9738/85/05/1397/06 $\$ 1.00$

Volume 75, May 1985, 1397-1402 the dog. In the cat and dog, the uppermost phrenic root mostly supplies the costal portion of the diaphragm, whereas the lowermost root mostly supplies the crural portion $(5,6)$. The phrenic nerve has $\sim 400$ axons in the rat and $\sim 1,600$ in the dog; in the $\operatorname{dog} \sim 65 \%$ are efferent and the remainder are afferent fibers $(7,8)$. The innervation ratio is $\sim 400$ muscle fibers/nerve axon in the rat (7), and by extrapolation from data in limb muscles (3), the ratio would probably be $\sim 2,500$ in humans. Proprioceptive receptors, especially muscle spindles, are relatively sparse in the diaphragm $(8,9)$. Thus, the diaphragm's ability to compensate for loads depends more on its intrinsic contractile properties than on neural reflex mechanisms.

The mean firing rate of phrenic motoneurones in the cat is $\sim 10$ impulses/second $(\mathrm{Hz})$ during quiet breathing and increases to $\sim 12 \mathrm{~Hz}$ with unobstructed $\mathrm{CO}_{2}$-induced hyperventilation. With airway occlusion, the firing rate rises as a function of inspiratory pressure to $\sim 30 \mathrm{~Hz}(10)$. The upper limit of motor unit discharge rates of human limb muscles during maximal voluntary contraction seems to be $30 \mathrm{~Hz}$ (11). Thus it is reasonable to suppose that frequencies of $10-30 \mathrm{~Hz}$ represent the physiologic range of intrinsic neural firing rates.

\section{Contractile properties}

The diaphragm, like other striated skeletal muscles, exhibits characteristic responses to stimulation. The speed of a single twitch contraction is measured as time to peak tension (TPT), and the rate of relaxation is measured as time required for peak tension to fall by half (one half relaxation time, $1 / 2 R T$ ). Diaphragms from small animals contract and relax more rapidly than those of larger species, and within a species TPT and $1 / 2$ RT are intermediate between those of fast and slow limb muscles. The values of TPT and $1 / 2$ RT obtained in vitro for canine diaphragm are 48 and $68 \mathrm{~ms}$, respectively (12), and values for human diaphragm are 88 and $117 \mathrm{~ms}$, respectively (2). In vivo, human diaphragm twitches evoked by phrenic nerve stimulation have TPT of $70 \mathrm{~ms}$ and $1 / 2 \mathrm{RT}$ of $67 \mathrm{~ms}$ (Bellemare, F., and J. J. Woods, personal communication).

The force of contraction is a function of three variables; the velocity of shortening (force-velocity response), the initial length of the muscle (force-length or length-tension response),

1. Abbreviations used in this paper: Edi, moving time average of diaphragmatic electrical activity; EMG, electromyogram; FRC, functional residual capacity; IRL, inspiratory resistive loading; L, diaphragm length; $L_{0}$, optimal resting length; Pdi, transdiaphragmatic pressure; $\bar{P}$ di breath, mean Pdi developed in one breath; Pdi max, maximal Pdi developed during a voluntary effort against a closed airway; $\mathbf{P}_{\mathbf{0}}$, maximal force per unit of diaphragm muscle cross-sectional area; $1 / 2$ $\mathrm{RT}$, one half relaxation time; $\mathrm{Ti} / \mathrm{Ttot}$, ratio of inspiratory to total breath duration; T/lim, time at which contractile force fails; TPT, time to peak tension; $\mathrm{Tr}$, time constant of the later monoexponential phase of relaxation; TTdi, tension-time index of diaphragm; V, lung volume. 
and the rate at which the muscle is stimulated (force-frequency response). The maximal velocity of shortening $\left(V_{\max }\right)$ is related to the speed of contraction because both are determined by myosin ATPase activity $(3,4)$. In vitro, $V_{\max }$ for the human diaphragm is 2.5 resting lengths per second, and for the rat diaphragm is 10 resting lengths per second, and among species the plot of $V_{\max }$ vs. 1/TPT is rectilinear (2).

Recently sonomicrometric techniques have been used to assess in vivo diaphragm length, and the extent and velocity of its shortening (13). The in situ $V_{\max }$ of canine diaphragm is $\sim 5$ resting lengths per second, a value that agrees well with that predicted from the in vitro relationship between $V_{\max }$ and 1/TPT (2). In panting dogs the diaphragm shortens at $\sim 25 \%$ $V_{\max }(14)$. The shortening velocity of the human diaphragm has not been measured, but an estimate can be made from the relationship between diaphragm length and lung volume $(\Delta \mathrm{L} / \Delta \mathrm{V})$ and inspiratory flow rate $(\Delta \mathrm{V} / \Delta t)$. Using roentgenographic and necropsy measurements of human diaphragm dimensions $(15,16)$, and maximal inspiratory flow volume data (17), $\Delta \mathrm{L} / \Delta \mathrm{V}$ is $\sim 1.25 \% \mathrm{~L}_{0}$ per percent total lung capacity, and $\Delta \mathrm{V} / \Delta t$ is $\sim 1$ total lung capacity per second. Combining these values gives an estimate of shortening velocity $(\Delta \mathrm{L} / \Delta t)$ of $1.25 \mathrm{~L}_{0} / \mathrm{s}$, or $50 \%$ of $V_{\max }$ for in vitro human diaphragm muscle (2). At this velocity, maximal stimulation would theoretically produce a contractile force that is $<20 \%$ of that in an isometric contraction (18). This is consistent with actual transdiaphragmatic pressure (Pdi) measured during maximal static and unobstructed inspiratory efforts (19), and supports Fenn's prediction (1) that inspiratory airflow is limited more by the ability of inspiratory muscles to develop pressure than by airway caliber.

The optimal resting length $\left(L_{0}\right)$ of in vitro muscle preparations is defined as the length at which peak twitch force is developed. Maximal tetanic stimulation at $\mathrm{L}_{0}$ yields maximal force $\left(P_{0}\right)$. The diaphragm begins to develop force at $0.5 L_{0}$, and active force is $\sim 50 \%$ of the maximal force per unit of diaphragm muscle cross-sectional area $\left(\mathrm{P}_{0}\right)$ at both 0.7 and $1.25 \mathrm{~L}_{0}(12)$. Beyond $\mathrm{L}_{0}$ muscles develop passive tension such that as active tension falls, total tension remains nearly constant. The in vitro force-length curves for diaphragm, when normalized for $L_{0}$ and $P_{0}$, are virtually identical to those of other muscles, and across species (12).

We estimated the force-length relationship of the in situ human diaphragm, using a roentgenographic technique to measure total diaphragm length (15), and data obtained at necropsy (16) to obtain the length of the muscular portion. Force was inferred from Pdi measured during maximal voluntary expiratory efforts. Data were obtained at four lung volumes from full expiration to full inspiration. The forcelength curve constructed from these values of Pdi and muscle length fits in vitro force-length curves quite well, and $\mathrm{L}_{0}$ seems to be at a lung volume below the resting volume (functional residual capacity, FRC). In the open-chest dog, the costal diaphragm contractile force and length responses to tetanic phrenic nerve stimulation have been measured directly (20). The resultant force-length curve also has the same characteristics as curves from in vitro muscle, and again $L_{0}$ seems to be at lung volume below FRC. In situ force-length characteristics of the costal and crural parts of the diaphragm have been determined in intact dogs, using sonomicrometry (21). In this study, $\mathrm{L}_{0}$ of the costal diaphragm lies at a lung volume above FRC, whereas the opposite was true for the crural diaphragm.
Thus, one or another part of the diaphragm is shorter than $\mathrm{L}_{0}$ at FRC. Because increasing lung volume shortens the diaphragm even more, it works at a progressively severe mechanical disadvantage at high lung volume.

Contractile force increases as a function of the rate at which a muscle is stimulated. The shape of the force-frequency curve is determined by contraction time and relaxation rate, because fusion of individual twitches occurs at lower tetanic stimulation rates in slow muscles. At $L_{0}, P t$ is $\sim 20-30 \% P_{0}$. At 10, 20, 30, and $50 \mathrm{~Hz}$ tetanic stimulation, the force responses are $\sim 30,75,85$, and $95 \% P_{0}$, respectively. Over the physiologic range of spontaneous nerve firing rates, $10-30 \mathrm{~Hz}$, the force output nearly triples. The shapes of the Pdi-frequency curves produced by percutaneous stimulation of the phrenic nerve in humans are virtually identical to shapes of in vitro force-frequency curves for diaphragm and limb skeletal muscles (22-24). At muscle lengths shorter than $L_{0}$, TPT and $1 / 2 R T$ decrease, and the ascending limb of the force-frequency curve is shifted to the right (25). For example, the force response to $20 \mathrm{~Hz}$ stimulation at $\mathrm{L}_{0}$ is matched by $50 \mathrm{~Hz}$ stimulation at $0.7 \mathrm{~L}_{0}$. Put another way, to obtain equivalent force at a shorter muscle length requires greater excitation, at levels that may not be physiologically attainable.

At $L_{0}$, the $P_{0}$ ranges from 1.3 to $2.8 \mathrm{~kg} / \mathrm{cm}^{2}$ for different species $(12,26,27)$. In hamsters, $P_{0}$ is not altered by exercise training, emphysema induced by intratracheal instillation of elastase, or simple semistarvation (26-28). The maximal force produced by tetanic stimulation of the in situ canine diaphragm is $1.5-2 \mathrm{~kg} / \mathrm{cm}^{2}(20)$. We used the shape and dimensions of the human diaphragm and body cavity to calculate its contractile force during a static inspiratory effort in which Pdi was $120 \mathrm{~cm} \mathrm{H}_{2} \mathrm{O}$ (29). Our estimate of $1.26 \mathrm{~kg} / \mathrm{cm}^{2}$ agrees well with the dog data, and with Fenn's calculations (1), but probably underestimates maximal force. It takes a combination of static inspiratory and expulsive efforts to maximally activate the diaphragm, as judged by responses to phrenic nerve stimulation superimposed on voluntary efforts (30). Under these circumstances Pdi is over $200 \mathrm{~cm} \mathrm{H}_{2} \mathrm{O}$, and maximal force would be $\sim 2 \mathrm{~kg} / \mathrm{cm}^{2}$.

\section{Mechanical actions in situ}

To understand the actions of the diaphragm on the respiratory system, it is necessary to appreciate its arrangement in situ (Fig. 1). Each hemidiaphragm is like an elliptical cylinder with a curved dome, with the top of the dome being somewhat flattened (31). The anterior wall of the cylindrical portion conforms to the costal margin, so the muscle fibers are longer posteriorly than anteriorly. Most of the muscle fibers are oriented parallel to the cranio-caudal body axis, with only the upper portions curved over the abdominal viscera.

Developmentally, anatomically, and functionally the diaphragm comprises two muscles $(6,23,33)$. The costal portion is thin and flat; its muscle fibers extend radially from the central tendon to insert on the rib cage. The crural portion is located next to the spine. It is thicker, and has a more complex criss-cross arrangement of fibers, especially around the aortic and esophageal hiatuses. Contraction of the diaphragm increases abdominal pressure and decreases pleural pressure. The increased abdominal pressure exerts an outward inspiratory force on the lower third of the rib cage, which is apposed to the diaphragm $(34,35)$. The upward pull of the costal diaphragm also causes the lower rib cage to expand. The negative pleural 


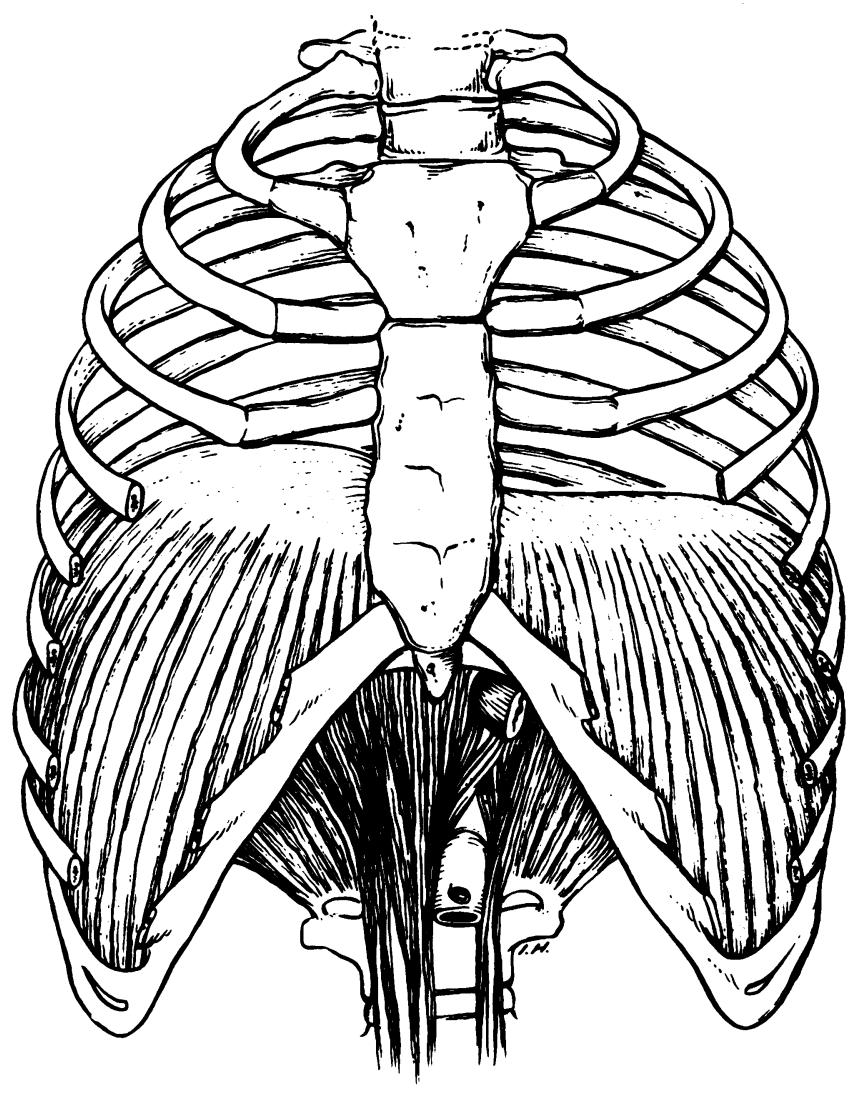

Figure 1. Representation of configuration of human diaphragm in situ, at full expiratory position. (Reproduced from Trans. Am. Clin. Climatol. Assoc., 1981, 83:200-208, by copyright permission of the American Clinical and Climatological Association.)

pressure inflates the lung, but also acts to decrease the diameter of the upper rib cage.

In an electric circuit analogue of muscle action, muscles (force generators) in series sum their forces but share a common length displacement. In contrast, muscles in electrical parallel exert a common force but sum their length displacements (36). In a mechanical model the series and parallel arrangements are just the opposite of these in the electric circuit analogue. At low and normal lung volumes the costal diaphragm is in electrical series with the crural diaphragm and with the intercostal and accessory inspiratory muscles, whereas the crural diaphragm is in electric parallel with the intercostal and accessory inspiratory muscles (36). These arrangements are altered at high lung volume, by a decrease in the area of apposition $(34,35)$ and by a change in the relationship between costal and crural diaphragm $(35,36)$.

In situ, as lung volume increases, the diaphragm shortens. Data from human and dog studies $(15,20)$ show a similar, slightly curvilinear relationship of diaphragm length to lung volume, with $\Delta \mathrm{L} / \Delta \mathrm{V}$ decreasing as lung volume increases. It is probable that there is a relatively constant relationship between diaphragm length, configuration, and lung volume under the conditions of those studies, although under other circumstances the diaphragm can be made to assume various other configurations at a given lung volume (34-36). Most or all of the reduction in Pdi after increasing lung volume can be explained by the force-length properties of the diaphragm
$(15,20)$. Diaphragm dome shape changes only slightly from full expiration to full inspiration (20), and the ratio of Pdi to directly measured contractile force remains constant until close to full inspiration (20). The constancy of the Pdi-force relationship results in part from its overall configurational characteristics (31).

\section{Fatigue}

Fatigue is defined as acute loss of contractile force after work. When sufficiently stressed, even the normal human diaphragm can become fatigued (37). The major determinants of fatigue are the force and duration of diaphragmatic contraction (38). Force is expressed as the ratio of the mean transdiaphragmatic pressure developed in one breath to the maximal transdiaphragmatic pressure developed during a voluntary effort against a closed airway ( $\overline{\mathrm{P} d i}$ breath/Pdi max) and duration is expressed as the ratio of inspiratory to total breath duration (Ti/Ttot). The product ( $\overline{\mathrm{Pdi}}$ breath/Pdi $\max ) \times(\mathrm{Ti} / \mathrm{Tt}$ tot) is referred to as the tension-time index (TTdi). During quiet breathing TTdi is $\sim 0.015$. With inspiratory resistive loading (IRL), TTdi increases, and fatigue occurs when TTdi exceeds 0.15 . The more that TTdi exceeds this critical value, the faster is the onset and the greater is the severity of fatigue. The same considerations hold for patients with chronic airflow obstruction, many of whom breathe close to the critical level of TTdi even at rest (39).

Diaphragmatic fatigue can be detected in various ways (Table I). The first four tests effectively represent a reduction in contractile force or Pdi in response to spontaneous or external stimulation. Failure to achieve a previously attainable Pdi maximum or targetted level of Pdi during IRL assumes that the level of voluntary neural activation is the same before and after the onset of fatigue, but the degree of activation is not quantified. In fact, the level of central neural activation decreases somewhat with fatigue (40). Simultaneous measurement of Pdi and the moving time average of diaphragmatic electrical activity (Edi) yields a Pdi/Edi ratio that falls when contractility is impaired, as for example in response to breathing $\mathrm{CO}_{2}$ enriched air (41), or with diaphragmatic fatigue induced by cardiogenic shock (42). In this test, Edi reflects central neural drive.

The force-frequency response curve also reflects a ratio of Pdi to activation, the latter being provided by external tetanic stimulation of the phrenic nerve. Again, fatigue reduces the Pdi response to simulation, the force-frequency response depending on the nature of the fatiguing effort. With severe IRL, force is lost both at low frequencies in the physiologic range (low frequency fatigue) and at high frequencies (high frequency fatigue) $(23,24)$. The decrement of force is $\sim 50 \%$ at $20 \mathrm{~Hz}$, and $\sim 10 \%$ at $100 \mathrm{~Hz}$ stimulation. Prolonged submaximal efforts produce low frequency but not high frequency fatigue $(23,43)$. Recovery from high frequency fatigue is relatively rapid, within $30 \mathrm{~min}$, but recovery from low frequency fatigue does not occur until after $1 \mathrm{~h}$ or more $(23,24,43)$.

The next two tests for diaphragmatic fatigue (Table I) reflect changes in muscle function that begin somewhat before contractile force decreases. Fatigued muscles relax more slowly than fresh muscles. This can be quantified by measuring the maximum relaxation rate, which varies with the force of contraction, or the time constant of the later monoexponential phase of relaxation ( $\mathrm{Tr}$ ). The latter is useful because it is independent of contractile force. Diaphragmatic fatigue induced 
Table I. Tests for Diaphragmatic Fatigue

\begin{tabular}{|c|c|c|}
\hline Test & Variable measured & Outcome in fatigue \\
\hline Pdi max & $\begin{array}{l}\text { Maximal transdiaphragmatic pressure (Pdi) with voluntary } \\
\text { effort }\end{array}$ & Decreases \\
\hline Targetted Pdi & Level of Pdi to be sustained against a ventilatory load & Cannot be maintained \\
\hline Pdi/Edi & Ratio of Pdi to Edi at various levels of voluntary effort & Decreases \\
\hline $\mathbf{T r}$ & $\begin{array}{l}\text { Time constant of monoexponential phase of relaxation after } \\
\text { abrupt cessation of contraction (measure from decrement in } \\
\text { Pdi) }\end{array}$ & Increases \\
\hline $\begin{array}{l}\text { Centroid frequency or } \\
\text { high/low ratio }\end{array}$ & $\begin{array}{l}\text { Distribution of power in diaphragm EMG as a function of } \\
\text { frequency content of EMG signal }\end{array}$ & Decreases only with high frequency fatigue \\
\hline TTdi & 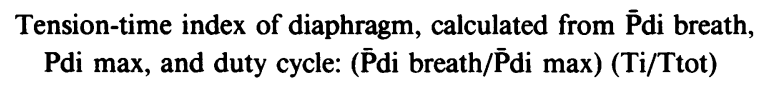 & $\begin{array}{l}\text { Increases with ventilatory load; fatigue } \\
\text { occurs when TTdi } \geq 0.15\end{array}$ \\
\hline Tlim & $\begin{array}{l}\text { Duration of breathing against a fatiguing load, indicated by } \\
\text { point when targetted Pdi can no longer be achieved }\end{array}$ & Time to fatigue decreases as TTdi increases \\
\hline
\end{tabular}

by severe IRL prolongs $\operatorname{Tr}$ substantially (44). This enables the diaphragm to maintain tension despite a reduction in central activation (40).

Muscle fatigue is associated with an alteration of the frequency content of the electromyogram (EMG), with power being redistributed from higher to lower frequencies. This is best quantified as a downward shift in the centroid frequency, measured using power spectral analysis $(45,46)$. A simple technique is to ascertain the ratio of power in a high frequency band to that in a low frequency band (high/low ratio). The high/low ratio of the diaphragm falls with fatigue $(47,48)$, with a time course and extent that closely match the prolongation of relaxation (44). To recapitulate, prolongation of relaxation and decrease in the high/low ratio precede overt fatigue, and the speed and magnitude of changes in these variables are strongly correlated with the intensity of the fatiguing effort $(44,48)$.

The reasons for the increase in relaxation time and decrease in the high/low ratio with fatigue are not fully understood. These phenomena may be related to the excitation-contraction coupling process, specifically to changes in muscle membrane function after alterations in muscle energy metabolism. Prolonged relaxation is associated with reductions in muscle phosphagen and $\mathrm{pH}$ (49), but relaxation can also be prolonged by reducing extracellular chloride concentration (50). Both low external chloride and $\mathrm{CO}_{2}$ acidosis increase muscle membrane resistance (51). The shift in the EMG power spectrum is unlikely to result from slowing the frequency of or synchronizing nerve firing rates, or to delay at the neuromuscular junction (52). It may be related to prolongation of sarcolemmal conduction velocity (48), although this point is debated (52). In any case, it is important to note that the power spectral shift in the EMG is present only when both high and low frequency fatigue are present. There is no change in centroid frequency or high/low ratio with low frequency fatigue alone, in either the diaphragm or limb muscles $(23,43)$.
The last test for diaphragmatic fatigue is indirect. When the TTdi exceeds its critical value, fatigue will develop at a rate that depends on how much TTdi exceeds the critical level, and how long that pattern of breathing is sustained (38). The relationship between the time at which contractile force fails $(\mathrm{T} / \mathrm{lim})$ and TTdi is $\mathrm{T} / \mathrm{lim}(\mathrm{min})=0.1$ (TTdi) $^{-3.6}$. This equation, which is valid only when TTdi is 0.15 or higher, predicts that the diaphragm will fatigue in $92 \mathrm{~min}$ at a TTdi of 0.15 , in 33 min at a TTdi of 0.2 , and in $8 \mathrm{~min}$ at a TTdi of 0.3 .

Current interest is focused on pharmacologic interventions to improve diaphragmatic contractility, and to prevent or reverse fatigue. The methyl xanthines aminophylline and caffeine improve contractility of human and canine diaphragms, as judged from increases in the Pdi/Edi ratio and forcefrequency response curves (53-56). The response is dosedependent (54), with $15-50 \%$ improvement at therapeutic doses $(53,55,56)$. The effect of aminophylline, but not of theophylline, is abolished by the calcium channel blocker verapamil (56). In the presence of diaphragmatic fatigue, aminophylline restores contractility, particularly in response to stimulation at $10-30 \mathrm{~Hz}(53,57)$. Isoproterenol and terbutaline in therapeutic doses have effects similar to those of methylxanthines on fatigued diaphragms $(57,58)$. However, terbutaline has no effect on contractility of the fresh diaphragm, again has most effect on low frequency fatigue, and its action is blocked by the $\beta$ blocker propanolol (58). These observations suggest that low frequency fatigue results from dysfunction of the excitation-coupling process, with impaired release of calcium from the sarcoplasmic reticulum, or altered calcium binding with troponin $(41,43,56,57)$. How methyl xanthines or sympathomimetic agents reverse the process is not entirely clear. Although the effects of aminophylline on the diaphragm have been confirmed in several laboratories, aminophylline has no effect on contractility of fresh or fatigued human adductor pollicis or sternomastoid muscles $(59,60)$. The reason for this discrepancy is not known. 


\section{The diaphragm in disease}

Inspiratory muscle strength is markedly reduced by weight loss and myopathy $(61,62)$, and when it falls below $30 \%$ normal hypercapnic respiratory failure is likely to occur (62). Hypercapnia impairs diaphragmatic contractility (41) and hypoxemia accelerates the onset of IRL-induced fatigue (63). In the hypoxic dog, blood flow to the diaphragm and oxygen extraction increase sufficiently to maintain aerobic work capacity until phrenic venous $\mathrm{PO}_{2}$ falls below 10-12 torr (64). Below that level, the diaphragm fails and the animals retain $\mathrm{CO}_{2}$. Severe hypotension also induces diaphragmatic fatigue and respiratory failure (42). Patients with isolated bilateral diaphragmatic paralysis live, but usually have chronic respiratory failure (65).

The reduction of maximal inspiratory pressure in human obstructive lung disease is partly a function of the diaphragm working at a disadvantageous length, partly a function of generalized muscle weakness, and is associated with hypercapnia (66). Muscle weakness renders the diaphragm more susceptible to fatigue because it increases the ratio ( $\overline{\mathrm{Pdi}}$ breath/Pdi max) by lowering the denominator, whereas the numerator is either unchanged or increased as in obstructive lung disease (39). Since $\mathrm{Ti} / \mathrm{T}$ tot remains near normal in obstructive lung disease, an increase in the ratio ( $\bar{P} d i$ breath/Pdi max) causes TTdi to approach the critical level for fatigue (39). Among patients undergoing attempts to discontinue mechanical ventilation, those who develop diaphragmatic fatigue display rapid, shallow breathing, abnormal movements of the chest and abdomen, and ultimately retain $\mathrm{CO}_{2}$ (67). Aminophylline prevents or ameliorates diaphragmatic fatigue after IRL, both in dogs and in patients with chronic obstructive pulmonary disease $(68,69)$.

Animal models of disease provide valuable insights into compensatory mechanisms that can occur in the diaphragm. With severe emphysema, the hamster diaphragm shifts its $L_{0}$ to a shorter absolute length by losing sarcomeres $(26,27,70)$. As a result, the length of remaining sarcomeres is closer to their $L_{0}$ despite hyperinflation of the lung. However, the magnitude of this compensation is small at degrees of hyperinflation that correspond to those in human obstructive lung disease. Emphysema does not alter diaphragmatic contractile properties or $P_{0}(26,27)$, but it can increase total contractile force of the whole diaphragm through hypertrophy (26). In sedentary hamsters emphysema causes selective atrophy of fast twitch fibers, which is prevented by concurrent exercise training (71). Emphysema alone and with training significantly increases the oxidative potential, and reduces glycolytic capacity of the hamster diaphragm (71).

Challenging problems exist for future research. One area concerns the relationship between in vitro and in vivo mechanical and contractile properties. Physiologic neural activation of a muscle involves stochastic rather than synchronous activation of motor units. It is conceivable that a given nerve firing frequency produces more force in the whole muscle than artificial stimulation at the same frequency, at less metabolic cost. The effects of muscle shortening mediated both by length per se and by the velocity of shortening on diaphragmatic blood flow, metabolism, and endurance remain to be established, particularly under physiologic states such as exercise.

The mechanisms of low frequency fatigue and its delayed recovery are still poorly understood. Most evidence supports the concept that these are mediated by altered excitation- contraction coupling, rather than impaired energy metabolism (22). Yet it is possible that during the period when energy supply is depleted there are structural or functional alterations in the various muscle membranes (sarcolemmal, sarcoplasmic reticular, and mitochondrial) that persist long after metabolism has returned to normal. The mechanisms underlying muscle fatigue might be protective, in the sense that contraction is inhibited before severe depletion of energy supply, so that irreversible structural damage is prevented. In this regard, the deleterious effects of infection, trauma, and neoplasm on muscle protein synthesis and degradation, and on muscle contractility are of great interest (72). All these issues are of paramount importance to the diaphragm as they concern factors that determine its function in disease. Contractility of the diaphragm is not absolutely essential for life, but it certainly is required for good health and the ability to carry out normal life activities.

\section{Acknowledgments}

I thank Dr. Gaspar Farkas for his critical review of this manuscript, and Mrs. Betty Edmondson for her help in preparing it.

This work was supported by National Institutes of Health grant HL 21500.

\section{References}

1. Fenn, W. O. 1963. A comparison of respiratory and skeletal muscles. In Houssay Memorial Papers. C. F. Cori, V. G. Foglia, L. F. Leloir, and S. Ochoa, editors. Elsevier Science Publishing Co., Inc., New York. 293-300.

2. Faulkner, J. A., L. C. Maxwell, G. L. Ruff, and T. P. White. 1979. Am. Rev. Respir. Dis. 119(2, part 2):89-92.

3. Buchthal, F., and H. Schmalbruch. 1980. Physiol. Rev. 60:90142.

4. Saltin B., and P. D. Gollnick. 1983. Skeletal muscle adaptability: significance for metabolism and performance. In Handbook of Physiology, L. D. Peachey, R. H. Adrian, and S. R. Geiger, editors. American Physiological Society, Waverly Press, Inc., Baltimore, MD. 551-631.

5. Sant'Ambrogio, G., D. T. Frazier, M. F. Wilson, and E. Agostoni. 1963. J. Appl. Physiol. 18:43-46.

6. DeTroyer, A., M. Sampson, S. Sigrist, and P. T. Macklem. 1982. J. Appl. Physiol. Respir. Environ. Exercise Physiol. 53:30-39. 439.

7. Krnjević, K., and R. Miledi. 1958. Am. J. Physiol. 140:427-

8. Landau, B. R., K. Abert, and T. S. Roberts. 1962. J. Comp. Neurol. 119:1-10.

9. Corda, M., C. V. Euler, and G. Lennerstrand. 1965. Am. J. Physiol. 178:161-177.

10. Iscoe, S., J. Dankoff, R. Migicovsky, and C. Polosa. 1976. Respir. Physiol. 26:113-128.

11. Bellemare, F., J. J. Woods, R. Johansson, and B. BiglandRitchie. 1983. J. Neurophysiol. (Bethesda). 50:1380-1392.

12. McCully, K. K., and J. A. Faulkner. 1983. J. Appl. Physiol. Respir. Environ. Exercise Physiol. 54:1681-1686.

13. Newman, S., J. Road, F. Bellemare, J. P. Clozel, C. M. Lavigne, and A. Grassino. 1984. J. Appl. Physiol. Respir. Environ. Exercise Physiol. 56:753-764.

14. Fitting, J. W., S. L. Newman, R. Znamirowski, A. Gueraty, and A. Grassino. 1984. Fed. Proc. 43:878. (Abstr.)

15. Braun, N. M. T., N. S. Arora, and D. F. Rochester. 1982. J. Appl. Physiol. Respir. Environ. Exercise Physiol. 53:405-412.

16. Arora, N. S., and D. F. Rochester. 1982. J. Appl. Physiol. Respir. Environ. Exercise Physiol. 52:64-70. 
17. Gal, T. J., and N. S. Arora. 1982. J. Appl. Physiol. Respir. Environ. Exercise Physiol. 52:57-63.

18. Rochester, D. F. (1982). Fatigue of the diaphragm. In Update: Pulmonary Diseases and Disorders. A. P. Fishman, editor. McGrawHill, Inc., New York. 89-100.

19. Milic-Emili, J., M. Orzalesi, C. D. Cook, and J. M. Turner. 1964. J. Appl. Physiol. 19:217-223.

20. Kim, M. J., W. S. Druz, J. Danon, W. Machnach, and J. T. Sharp. 1976. J. Appl. Physiol. 41:369-382.

21. Road, J. D., J. P. Derenne, and A. Grassino. 1984. Fed. Proc. 43:530. (Abstr.)

22. Edwards, R. H. T. 1979. Am. Rev. Respir. Dis. 119(2, part 2): 81-84.

23. Moxham, J., A. J. R. Morris, S. G. Spiro, R. H. T. Edwards, and M. Green. 1981. Thorax. 36:164-168.

24. Aubier, M., G. Farkas, A. DeTroyer, R. Mozes, and C. Roussos. 1981. J. Appl. Physiol. Respir. Environ. Exercise Physiol. 50:538-544. 25. Farkas, G. A., and C. Roussos. 1984. Am. Rev. Respir. Dis. 130:434-438.

26. Supinski, G. S., and S. G. Kelsen. 1982. J. Clin. Invest. 70: 978-988.

27. Farkas, G. A., and C. Roussos. 1982. J. Appl. Physiol. Respir. Environ. Exercise Physiol. 53:1263-1272.

28. Kelsen, S. G., M. Ference, and S. Kapoor. 1985. J. Appl. Physiol. Respir. Environ. Exercise Physiol. In press.

29. Rochester, D. F., N. S. Arora, and N. M. T. Braun. 1981 Trans. Am. Clin. Climatol. Assoc. 83:200-208.

30. Bellemare, F., and B. Bigland-Ritchie. 1984. Respir. Physiol. 58:263-278.

31. Whitelaw, W. A., L. E. Hajdo, and J. A. Wallace. 1983. J. Appl. Physiol. Respir. Environ. Exercise Physiol. 55:1899-1905.

32. DeTroyer, A., M. Sampson, S. Sigrist, and P. T. Macklem. 1981. Science (Wash. DC). 213:237-238.

33. Decramer, M., A. DeTroyer, S. Kelly, and P. T. Macklem 1984. J. Appl. Physiol. Respir. Environ. Exercise Physiol. 56(6):14841490.

34. Mead, J., and S. H. Loring. 1982. J. Appl. Physiol. Respir Environ. Exercise Physiol. 53:750-755.

35. Loring, S. H., and J. Mead. 1982. J. Appl. Physiol. Respir Environ. Physiol. 53:756-760.

36. Macklem, P. T., D. M. Macklem, and A. DeTroyer. 1983. J. Appl. Physiol. Respir. Environ. Exercise Physiol. 55:547-557.

37. Roussos, C., and P. T. Macklem. 1977. J. Appl. Physiol. Respir Environ. Exercise Physiol. 43:189-197.

38. Bellemare, F., and A. Grassino. 1982. J. Appl. Physiol. Respir Environ. Exercise Physiol. 53:1190-1195.

39. Bellemare, F., and A. Grassino. 1983. J. Appl. Physiol. Respir Environ. Exercise Physiol. 55:8-15.

40. Bellemare, F., and B. Bigland-Ritchie. 1984. Am. Rev. Respir. Dis. 129:268a. (Abstr.)

41. Juan, G., P. Calverly, C. Talamo, J. Schnader, and Ch. Roussos. 1984. N. Engl. J. Med. 310:874-879.

42. Aubier, M., T. Trippenbach, and C. Roussos. 1981. J. Appl. Physiol. Respir. Environ. Exercise Physiol. 51:499-508.

43. Moxham, J., R. H. T. Edwards, M. Aubier, A. DeTroyer, G. Farkas, P. T. Macklem, and C. Roussos. 1982. J. Appl. Physiol. Respir. Environ. Exercise Physiol. 53:1094-1099.

44. Esau, S. A., F. Bellemare, A. Grassino, S. Permutt, C. Roussos, and R. L. Pardy. 1983. J. Appl. Physiol. Respir. Environ. Exercise Physiol. 54:1353-1360.
45. Schweitzer, T. W., J. W. Fitzgerald, J. A. Bowden, and P. Lynne-Davies. 1979. J. Appl. Physiol. Respir. Environ. Exercise Physiol. 46:152-165.

46. Aldrich, T. K., J. M. Adams, N. S. Arora, and D. F. Rochester. 1983. J. Appl. Physiol. Respir. Environ. Exercise Physiol. 54:15791584.

47. Gross, D., A. Grassino, W. R. D. Ross, and P. T. Macklem. 1979. J. Appl. Physiol. Respir. Environ. Exercise Physiol. 46:1-7.

48. Bellemare, F., and A. Grassino. 1982. J. Appl. Physiol. Respir. Environ. Exercise Physiol. 53:1196-1206.

49. Dawson, M. J., D. G. Gadian, and D. R. Wilkie. 1980. J. Physiol. (Lond.) 299:465-484.

50. Esau, S. A. 1984. Am. Rev. Respir. Dis. 129:271a. (Abstr.)

51. Sperelakis, N. 1969. Am. J. Physiol. 217:1069-1075.

52. Kranz, H., A. M. Williams, J. Cassell, D. J. Caddy, and R. B. Silberstein. 1983. J. Appl. Physiol. Respir. Environ. Exercise Physiol. 55:392-399.

53. Aubier, M., A. DeTroyer, M. Sampson, P. T. Macklem, and C. Roussos. 1981. N. Engl. J. Med. 305:249-252

54. Sigrist, S., D. Thomas, S. Howell, and C. Roussos. 1982. Am. Rev. Respir. Dis. 126:46-50.

55. Supinski, G. S., E. C. Deal, Jr., and S. G. Kelsen. 1984. Am. Rev. Respir. Dis. 130:429-433.

56. Aubier, M., D. Murciano, N. Viires, Y. Lecocguic, and R. Pariente. 1983. J. Appl. Physiol. Respir. Environ. Exercise Physiol. 53: 460-464.

57. Howell, S., and C. Roussos. 1984. Am. Rev. Respir. Dis. 129: 118-124.

58. Aubier, M., N. Viires, D. Murciano, G. Medrano, Y. Lecocguic, and R. Pariente. 1984. J. Appl. Physiol. Respir. Environ. Exercise Physiol. 56:922-929.

59. Wiles, C. M., J. Moxham, D. Newham, and R. H. T. Edwards. 1983. Clin. Sci. (Lond.) 64:547-550.

60. Lewis, M. I., M. J. Belman, and G. C. Sieck. 1984. Chest. 86 803. (Abstr.)

61. Arora, N. S., and D. F. Rochester. 1982. Am. Rev. Respir. Dis. 126:5-8.

62. Braun, N. M. T., N. S. Arora, and D. F. Rochester. 1983. Thorax. 38:616-623.

63. Jardim, J., G. Farkas, C. Prefaut, D. Thomas, P. T. Macklem, and Ch. Roussos. 1981. Am. Rev. Respir. Dis. 124:274-279.

64. Reid, M. B., and R. L. Johnson, Jr. 1983. J. Appl. Physiol. Respir. Environ. Exercise Physiol. 54:763-772.

65. Kreitzer, S. M., N. T. Feldman, N. A. Saunders, and R. H. Ingram. 1978. Am. J. Med. 65:89-95.

66. Rochester, D. F., N. M. T. Braun, and N. S. Arora. 1979. Am. Rev. Respir. Dis. 119(2, part 2):151-154.

67. Cohen, C. A., G. Zagelbaum, D. Gross, Ch. Roussos, and P. T. Macklem. 1982. Am. J. Med. 73:308-316.

68. Viires, N., M. Aubier, D. Murciano, B. Fleury, C. Talamo, and R. Pariente. 1984. Am. Rev. Respir. Dis. 129:396-402.

69. Murciano, D., M. Aubier, Y. Lecocguic, and R. Pariente. 1984. N. Engl. J. Med. 311:349-353.

70. Farkas, G. A., and Ch. Roussos. 1983. J. Appl. Physiol. Respir. Environ. Exercise Physiol. 54:1635-1640.

71. Farkas, G. A., and Ch. Roussos. 1984. J. Clin. Invest. 74:12141220.

72. Ruff, R. L., and D. Secrist. 1984. J. Clin. Invest. 73:14831486. 